RUB-TPII-03/08

\title{
New vistas of the meson structure in QCD from low to high energies
}

\author{
N. G. Stefanis ${ }^{a *}$

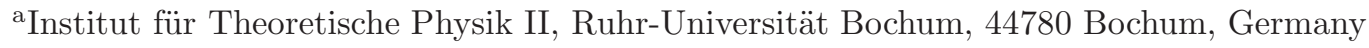

This talk presents issues pertaining to the quark structure of the pion within QCD, both from the theoretical and from the experimental point of view. We review and discuss the pion-photon transition form factor and the pion's electromagnetic form factor vs. corresponding experimental data from the CLEO Collaboration and the JLab. We also examine the extent to which recent high-precision lattice computations of the second moment of the pion's distribution amplitude conform with theoretical models. Finally, we include predictions for the azimuthal asymmetry of the $\mu^{+}$distribution in the polarized $\mu$-pair-induced DY production employing various pion distribution amplitudes.

\section{Introduction}

Understanding the quark structure of the lightest meson, the pion,- - the prototype for a meson bound state - is arguably one of the most basic, albeit challenging, questions QCD is still facing even after decades of intense investigations. Integrating over transverse momenta up to some resolution scale $\mu_{0}^{2}$, one gets from the pion's light-cone wave function the pion's leading twist-2 distribution amplitude (DA), $\varphi_{\pi}\left(x, \mu_{0}^{2}\right)$, defined in terms of a nonlocal axial current:

$$
\begin{aligned}
& \left.\left\langle 0\left|\bar{d}(z) \gamma^{\mu} \gamma_{5} \mathcal{C}(z, 0) u(0)\right| \pi(P)\right\rangle\right|_{z^{2}=0}= \\
& i f_{\pi} P^{\mu} \int_{0}^{1} d x \mathrm{e}^{i x(z \cdot P)} \varphi_{\pi}^{\mathrm{Tw}-2}\left(x, \mu_{0}^{2}\right),
\end{aligned}
$$

where $x(\bar{x} \equiv 1-x)$ is the longitudinal momentum fraction carried by the valence quark (antiquark) in the pion and the path-ordered exponential $\mathcal{C}(z, 0)=\mathcal{P} \exp \left[-i g \int_{0}^{z} d y^{\mu} t^{a} A_{\mu}^{a}(y)\right]$ ensures gauge invariance. Appealing to its renormalization-group properties [12], we can expand $\varphi_{\pi}^{\mathrm{Tw}-2}\left(x, \mu_{0}^{2}\right)$ in terms of its one-loop eigenfunctions, alias the Gegenbauer polynomials, to

\footnotetext{
*Talk presented at International Workshop on $e^{+} e^{-}$col-
} lisions from Phi to Psi, Frascati, Italy, 7-10 April 2008.

$$
\begin{aligned}
& \text { obtain } \\
& \begin{aligned}
\varphi^{\mathrm{Tw}-2}\left(x ; \mu_{0}^{2}\right)= & \varphi^{\mathrm{as}}(x)\left[1+a_{2}\left(\mu_{0}^{2}\right) C_{2}^{3 / 2}(2 x-1)\right. \\
& \left.+a_{4}\left(\mu_{0}^{2}\right) C_{4}^{3 / 2}(2 x-1)\right]+\ldots(2)
\end{aligned}
\end{aligned}
$$

Here $\varphi^{\text {as }}(x)=6 x \bar{x}$ is the asymptotic pion DA and by virtue of the leptonic decay $\pi \rightarrow \mu^{+} \nu_{\mu}$ one has the normalization $\int_{0}^{1} d x \varphi_{\pi}^{\mathrm{Tw}-2}\left(x, \mu_{0}^{2}\right)=$ 1. Relying only upon the first two Gegenbauer coefficients, Eq. (2) can yield distinct profiles, as shown in Fig. 1. We will see in the next section how two-photon processes can be used to resolve pion's dilemma and reveal its parton substructure in agreement with the experimental data and the latest lattice calculations.

\section{Pion DA, CLEO data, and lattice esti- mates}

The entire nonperturbative content of the pion DA is encoded in the expansion coefficients $a_{n}\left(\mu^{2}\right)$ which in turn can be derived from the moments

$\left\langle\xi^{N}\right\rangle_{\pi} \equiv \int_{0}^{1} d x(2 x-1)^{N} \varphi_{\pi}(x)$,

where $\xi \equiv 2 x-1$, that decrease with increasing polynomial order $N$ to $0:\left\langle\xi^{N}\right\rangle_{\pi} \rightarrow[3 /(N+$ 


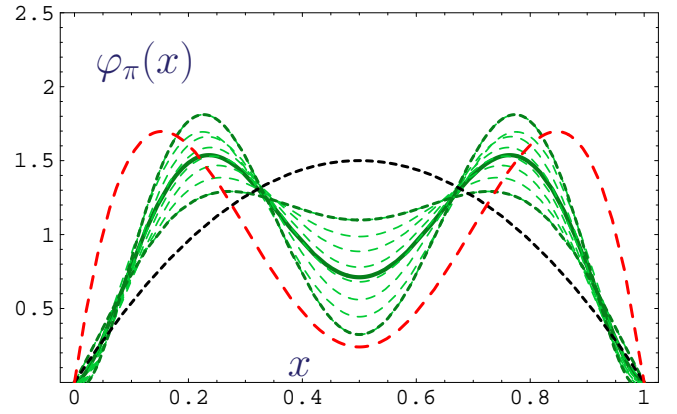

Figure 1. Pion's dilemma: to B (Bactrian camel) dashed line (CZ) 3 and BMS "bunch" 4-or to D (Dromedary camel) - dotted line (asymptotic DA)? All curves are shown at the normalization scale $\mu^{2}=1 \mathrm{GeV}^{2}$.

1) $(N+3)$ ]. The evolution behavior of $a_{n}\left(\mu^{2}\right)$ is controlled by the ERBL equation [1/2 (for a pedagogical exposition, see [5]). In our approach [4] we have determined $a_{n}$ at a normalization scale $\mu_{0}^{2}=1.35 \mathrm{GeV}^{2}$ with the help of QCD sum rules with nonlocal condensates 617. There are alternative approaches, based, for instance, on local QCD sum rules [3], instantons 8910, etc.

The predictive power of these theoretical approaches was challenged by the high-precision data of the CLEO Collaboration on the pionphoton transition 11, in which one photon has a large virtuality $Q^{2}$, while the other is nearly on shell. Kroll and Raulfs [12 were the first to show that the popular CZ pion DA was overshooting these data considerably. Other analyses, having recourse to light-cone sum rules with a spectral density obtained in the standard factorization scheme of perturbative QCD in leading order (LO) 13 and next-to-leading-order (NLO) [14 15]16]17, followed, which established the following facts (consult Fig. 2 drawn at the main scale, $\mu_{\text {CLEO }}^{2}=(2.4 \mathrm{GeV})^{2}$, probed in the CLEO experiment): (i) The $\mathrm{CZ}$ pion $\mathrm{DA}$ is outside the $4 \sigma$ error ellipse of the CLEO data. (ii) The asymptotic pion DA is outside the $3 \sigma$ ellipse, while (iii) other proposed models close to that are at least $2 \sigma$ 's off. (iv) The "bunch" of pion DAs derived from nonlocal QCD sum rules mostly overlaps with the $1 \sigma$ error ellipse, with the BMS model DA being entirely inside.

The most recent lattice calculations [18] (larger band bounded by dashed lines) and [19] (narrower band within solid lines) in Fig. 2 support and en-

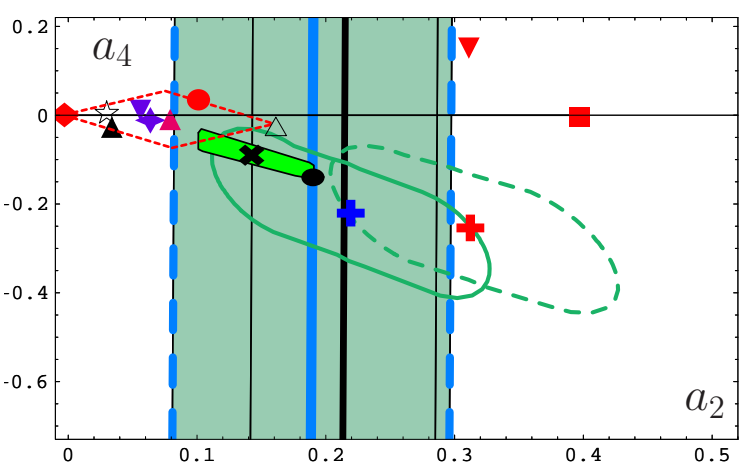

Figure 2. Comparison at $\mu_{\text {CLEO }}^{2}$ of various pion DA models with the CLEO data in terms of the $1 \sigma$ ellipse (solid line) and recent lattice simulations, denoted by vertical dashed lines 18 and solid ones 19. The symbols mark the models discussed in Tables 1 and 2 The slanted shaded rectangle represents the BMS DA "bunch" 4. The dashed $1 \sigma$ ellipse corresponds to the inclusion of the twist4 contribution to the pion DA via renormalons [17. The red bullet denotes the prediction derived from the holographic model based on AdS/CFT duality (see last section).

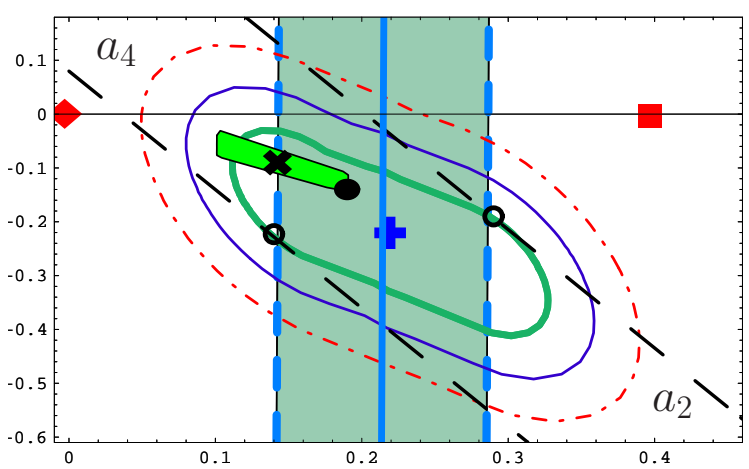

Figure 3. Range of values of $\left\langle\xi^{4}\right\rangle_{\pi}\left(\mu_{\text {CLEO }}^{2}\right)$ (slanted broken lines) intersecting with the $1 \sigma$ error ellipse (inner solid line) of the CLEO data and the latest lattice results on $a_{2}$ of the UKQCD-RBC Collaboration [19] (vertical shaded band).

hance these findings as regards the range of values of the first Gegenbauer coefficient $a_{2}$. First, as one sees from this figure, they rather disfavor a relatively large twist- 4 contribution to the pion DA, estimated with the help of renormalons [17. (dashed $1 \sigma$ error ellipse). Second, the latest calculation [19, with even smaller uncertainties than 18, indicates a trend further away from the asymptotic DA, but still in compliance with the BMS results.

Tables 1 and 2 show the values of the second and the fourth moment of various pion DAs at 
the lattice reference scale $\mu_{\text {Lat }}^{2}=4 \mathrm{GeV}^{2}$.

Table 1

Predictions for $\left\langle\xi^{2}\right\rangle_{\pi}$ from various models (methods) after NLO evolution from their proprietary normalization point to the scale $\mu_{\text {Lat }}^{2}=4 \mathrm{GeV}^{2}$.

\begin{tabular}{|c|c|c|c|}
\hline Source & Min & Mid & $\operatorname{Max}$ \\
\hline UKQCD/RBC [19] & 0.252 & 0.278 & 0.304 \\
\hline QCDSF/UKQCD 18 & 0.230 & 0.269 & 0.308 \\
\hline 20 & 0.232 & 0.285 & 0.338 \\
\hline 15]16 $(1 \sigma$ CLEO $) \boldsymbol{廿}$ & 0.240 & 0.280 & 0.320 \\
\hline [4] $*$ & 0.233 & 0.248 & 0.264 \\
\hline 14 ○ & 0.233 & 0.269 & 0.305 \\
\hline$[10]$ & - & 0.223 & - \\
\hline 9 $\triangle$ & - & 0.212 & - \\
\hline [8] & - & 0.211 & - \\
\hline $\begin{array}{ll}21 & \triangle\end{array}$ & - & 0.259 & - \\
\hline 22$] \Delta$ & - & 0.229 & - \\
\hline 23$] \nabla$ & - & 0.312 & - \\
\hline 3] & - & 0.344 & - \\
\hline Asy $\bullet$ & - & 0.199 & - \\
\hline $\mathrm{AdS} / \mathrm{QCD} O$ & - & 0.237 & - \\
\hline
\end{tabular}

In Fig. 3, we determine the range of values of the fourth moment $\left\langle\xi^{4}\right\rangle_{\pi}\left(a_{2}, a_{4} \mid \mu^{2}\right)$ (denoted by the slanted broken lines) that simultaneously fulfill the CLEO data and the lattice constraints on $\left\langle\xi^{2}\right\rangle_{\pi}$ from [19]. For each error ellipse, there is some overlap with the vertical $a_{2}$-band estimated in [19. For the maximum and minimum of $\left\langle\xi^{4}\right\rangle_{\pi}$ with respect to the $1 \sigma$ error ellipse, we find

$0.095 \leq\left\langle\xi^{4}\right\rangle_{\pi}\left(\mu_{\text {Lat }}^{2}\right) \leq 0.134$,

corresponding to $\left(a_{2}^{\min }=0.149, a_{4}^{\min }=-0.241\right)$ and $\left(a_{2}^{\max }=0.307, a_{4}^{\max }=-0.205\right)$. [Recall that $\left\langle\xi^{4}\right\rangle_{\pi}^{\text {asy }}=3 / 35$.] It happens that these values almost coincide with the intersection points (open circles) of the $1 \sigma$ error ellipse and the boundaries of the vertical band of the $a_{2}$ lattice constraints.

Figure 4 displays various theoretical predictions for $Q^{2} F_{\gamma^{*} \gamma \rightarrow \pi}\left(Q^{2}\right)$ vs. $Q^{2}$ in comparison with the CELLO (diamonds, 24]) and the CLEO (triangles, [11) experimental data, evaluated with the twist-4 parameter value $\delta_{\mathrm{Tw}-4}^{2}=$ $0.19 \mathrm{GeV}^{2}$ [1516]17. The other curves shown
Table 2

Predictions for $\left\langle\xi^{4}\right\rangle_{\pi}$ from various models (methods) after NLO evolution from their proprietary normalization point to the scale $\mu_{\text {Lat }}^{2}=4 \mathrm{GeV}^{2}$.

\begin{tabular}{|c|c|c|c|}
\hline Source & Min & Mid & $\operatorname{Max}$ \\
\hline $1516(1 \sigma$ CLEO $)$ & 0.066 & 0.115 & 0.162 \\
\hline 4 & 0.105 & 0.108 & 0.113 \\
\hline 14 & 0.082 & 0.116 & 0.151 \\
\hline 10 & - & 0.10 & - \\
\hline 9 & — & 0.091 & - \\
\hline 8 & - & 0.094 & - \\
\hline 21 & - & 0.121 & - \\
\hline 22 & - & 0.104 & - \\
\hline 23 & — & 0.178 & - \\
\hline 3 & - & 0.181 & - \\
\hline Asy & - & 0.085 & - \\
\hline $\mathrm{AdS} / \mathrm{QCD}$ & - & 0.114 & - \\
\hline
\end{tabular}

correspond to selected pion DAs: the asymptotic DA $\varphi_{\text {as }}$ (lower dashed line), $\varphi_{\mathrm{CZ}}$ (upper dashed line) [3, and two instanton-based models, viz., 8. (dotted line) and 10 (dash-dotted line). An important observation from this figure is that the shaded strip, which corresponds to the BMS "bunch" 4, becomes narrower at lower scales around $1 \mathrm{GeV}^{2}$. The reason is that at such low scales, the form factor is dominated by its twist- 4 contribution, while the leading twist-2 part dies out. Moreover, Fig. 4 makes it clear that the low- $Q^{2}$ CELLO data 24] exclude $\varphi_{\pi}^{\text {asy }}$ and clones, while the high- $Q^{2}$ CLEO data [11] rule out $\varphi_{\pi}^{\mathrm{CZ}}$. Figure 5 gives an illustration of the partial contributions to $Q^{2} F^{\gamma^{*} \gamma \pi}$, originating from different sources at $\mu_{\mathrm{CLEO}}^{2}$. A comprehensive account of these effects can be found in [17.

\section{Electromagnetic pion form factor}

The analysis of the pion's electromagnetic form factor involves on the nonperturbative side the BMS "bunch" of pion DAs in comparison with $\varphi_{\pi}^{\text {asy }}$ and $\varphi_{\pi}^{\mathrm{CZ}}$. On the perturbative side, a theoretical scheme is used, which consists of expanding the form factor in terms of analytic images of the strong running coupling and its powers up to the NLO. The basis of this approach develops 


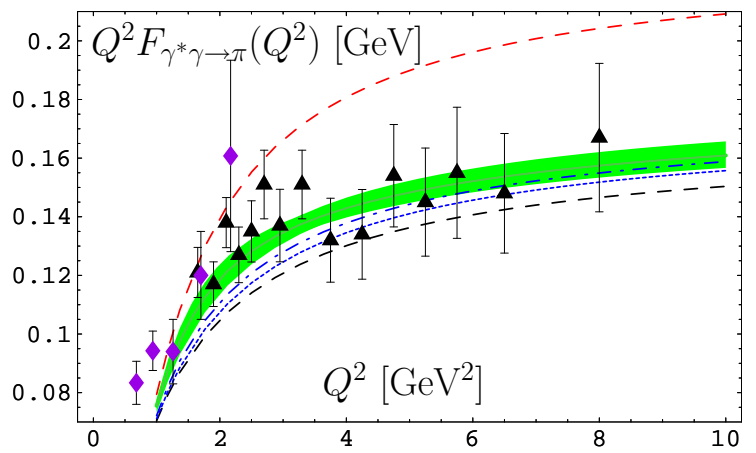

Figure 4. Predictions for $Q^{2} F_{\gamma^{*} \gamma \rightarrow \pi}\left(Q^{2}\right)$ vs. $Q^{2}$ in comparison with available experimental data (see text).

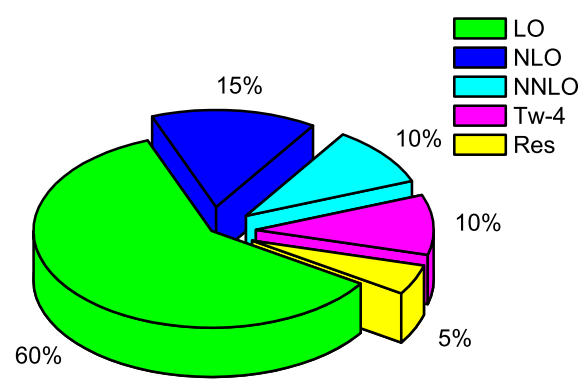

Figure 5. Contributions to $Q^{2} F^{\gamma^{*} \gamma \pi}$ at the typical scale $\mu^{2}=5.76 \mathrm{GeV}^{2}$ of the CLEO data [1]. The next-tonext-to-leading order (NNLO) estimate is based on 25], whereas the uncertainty owing to the (Res)onance model used, was discussed in [13].

from [26] and can account for more than one hard scale by incorporating into the "analytization" procedure all terms that contribute to the spectral density of the amplitude [27. The bedrock of the approach was developed in [27/28/29/30]31/32 and its application to $F_{\pi}^{\mathrm{NLO}}\left(Q^{2}\right)$ was considered in 3334 (see also 35). The results are displayed in Fig. 6 in comparison with experimental data. Note that the quantity shown comprises a soft non-factorizing contribution which dominates at currently accessible momentum transfers 33 .

The main characteristics of this approach are (i) a strongly reduced sensitivity on the renormalization and the factorization scale, (ii) an undiminished quality of precision in adopting different choices of renormalization schemes and scale settings, virtually eliminating the dependence variations from scheme to scheme and scale to scale [34. (iii) Another important finding is that, within such an analytic approach, the form-factor

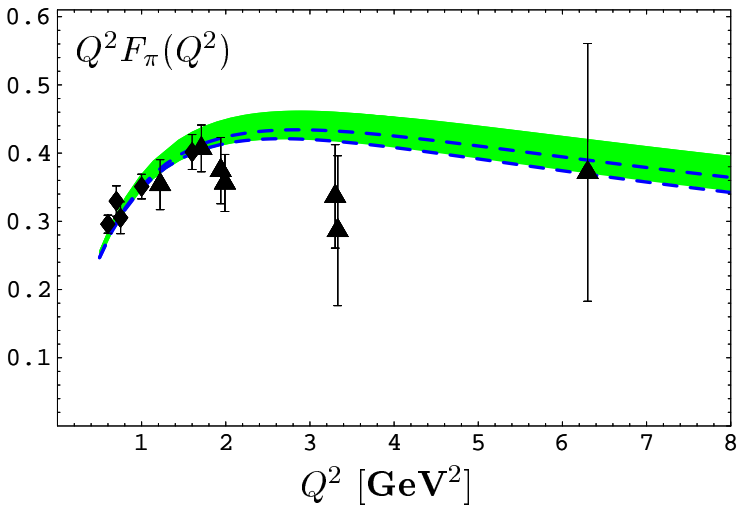

Figure 6. Predictions for the scaled pion form factor calculated with the BMS "bunch" (shaded strip) 4 in NLO QCD analytic perturbation theory [33. The dashed lines inside the strip restrict the area of predictions accessible to the asymptotic pion DA. The experimental data are taken from 36] (diamonds) and 37] (triangles).

predictions (shaded strip in Fig. 6) turn out to be very close to that computed with $\varphi_{\pi}^{\text {asy }}$, albeit the underlying pion DA profiles are very different. This proved that what really matters is the behavior of the pion DA at the endpoints $x \rightarrow 0,1$.

\section{Conclusions}

We have shown that the CLEO data on $F^{\gamma^{*} \gamma \pi}$ pose a veto to a variety of proposed models for the pion DA (see Fig. 2) and favor an endpointsuppressed "B"-shaped pion DA - a Bactrian "camelino", the endpoint suppression being provided by the vacuum nonlocality $\lambda_{q}^{2}=0.4 \mathrm{GeV}^{2}$. The crucial 'missing link' is the determination of $\left\langle\xi^{4}\right\rangle_{\pi}$ on the lattice. A value within the range $[0.095-0.134](2 \mathrm{GeV})$, extracted from the CLEO data in conjunction with the lattice constraints on $\left\langle\xi^{2}\right\rangle_{\pi}$ from [19] (cf. Fig. 3), would validate the claim that the pion DA is BMS-like.

The Drell-Yan process $\pi^{-} N \rightarrow \mu^{+} \mu^{-} X$ for lepton-pair production with a large invariant mass $Q^{2}$ provides an additional useful tool to probe and test different pion DAs in terms of azimuthal asymmetries, as Fig. 7 illustrates for the case of the kinematic variable $\mu$. Overall, a rather good agreement of the BMS "bunch", derived from nonlocal QCD sum rules [4, with 


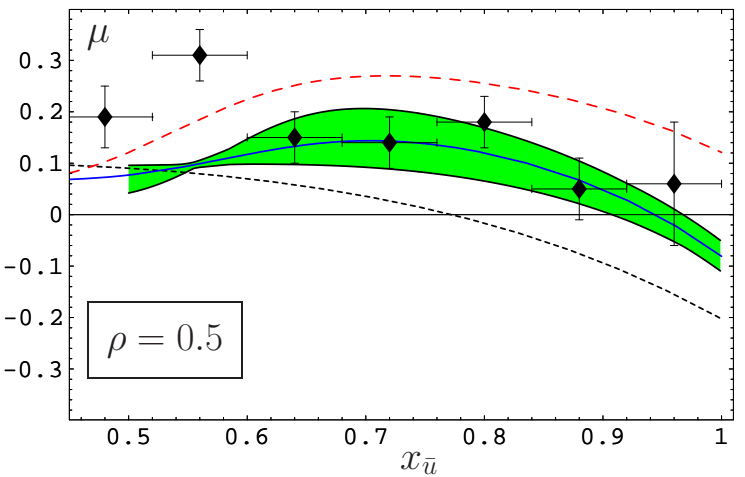

Figure 7. Results for the angular distribution parameter $\mu$ as a function of $x_{\bar{u}} \equiv x_{\pi}$ for $\rho \equiv Q_{T} / Q=0.5$. The shaded strip contains the results for the BMS "bunch" of DAs 44. The solid line corresponds to the BMS model, the dotted solid denotes the result for $\varphi_{\pi}^{\text {asy }}$, and the (red) dashed line is the prediction for the endpoint-dominated CZ DA [3]. One-loop evolution of the pion DAs to each measured $Q^{2}$ value is included (data taken from [38]).

available data was found [39], though the existing data cannot single out a particular model. In this respect, the planned COMPASS experiment may be of significant relevance.

\section{Section added: Holographic dual of QCD}

In this extra section, contained only in the arXiv version of this paper, we provide some predictions from the AdS/QCD approach.

In the holographic model of Brodsky and de Téramond (see, e.g., 40]), the pion DA has the form

$\varphi(x)_{\pi}^{\mathrm{hol}} \sim \sqrt{x(1-x)}$

with the normalization

$\int_{0}^{1} d x \varphi(x)_{\pi}^{\mathrm{hol}}=B(3 / 2,3 / 2)=\frac{\pi}{8}$

and moments given by

$\left\langle\xi^{2 n}\right\rangle_{\pi}^{\text {hol }}=\frac{1}{4} \frac{B(3 / 2,(2 n+1) / 2)}{B(3 / 2,3 / 2)}$,

where $B(x, y)$ is the Euler Beta function. Taking the first derivative of $\varphi(x)_{\pi}^{\text {hol }}, \sim \frac{1}{\sqrt{x}}$, one realizes that this DA has its endpoints strongly enhanced, even relative to the asymptotic DA, let alone to the BMS DA (cf. Fig. 11). Note that also the twist-4 DA contribution, extracted from the holographic model by Agaev and Gomshi Nobary [41, shows endpoint enhancement, although this effect is milder compared to the twist-2 DA case. It is interesting to recall at this point that Eq. (5D) was considered long ago by Mikhailov and Radyushkin in an attempt to reconstruct the pion DA from its first few moments within the context of QCD sum rules with nonlocal condensates [6].

The values of the moments $\left\langle\xi^{2}\right\rangle_{\pi}$ and $\left\langle\xi^{4}\right\rangle_{\pi}$ of the holographic model have been inserted in Tables 1 and 2 respectively, (last entry in each of these tables) after NLO evolution from their initial values $\left\langle\xi^{2}\right\rangle_{\pi}=1 / 4$ and $\left\langle\xi^{4}\right\rangle_{\pi}=1 / 8$ to the lattice scale $\mu_{\text {lat }}^{2}$ (with associated Gegenbauer coefficients $a_{2}^{\text {hol }}\left(\mu_{\text {lat }}^{2}\right)=0.107$ and $\left.a_{4}^{\text {hol }}\left(\mu_{\text {lat }}^{2}\right)=0.038\right)$.

The holographic model is also included in Fig. 2 in comparison with other theoretical models and confronted with the CLEO data [1], in parallel with the two most recent and precise lattice calculations [18]19. As for the moments above, we have assumed that the holographic DA, given by Eq. (5) is normalized at the scale $\mu^{2}=1 \mathrm{GeV}^{2}$ with the Gegenbauer coefficients $a_{2}^{\text {hol }}\left(\mu^{2}=1 \mathrm{GeV}^{2}\right)=7 / 48$ and $a_{4}^{\text {hol }}\left(\mu^{2}=\right.$ $\left.1 \mathrm{GeV}^{2}\right)=11 / 192$. We then performed a twoloop evolution to the scale $\mu_{\text {CLEO }}^{2}$ and found

$a_{2}^{\text {hol }}\left(\mu_{\text {CLEO }}^{2}\right)=0.101, \quad a_{4}^{\text {hol }}\left(\mu_{\text {CLEO }}^{2}\right)=0.03$.

The position of the holographic model is marked in Fig. 2 by a red bullet and turns out to be (cf. Fig. (3) just inside the border of the $2 \sigma$ ellipse of the CLEO data, being also inside the predictions' range of the QCDSF/UKQCD Collaboration, but outside the limits on $a_{2}$ determined by the UKQCD-RBC07 Collaboration.

Predictions for the spacelike (and timelike) pion form factor, derived from AdS/QCD, can be found, for example, in [4014142]43; they are not addressed here.

\section{Acknowledgments}

I wish to thank A.P. Bakulev for collaboration and S.V. Mikhailov for collaboration and technical help in preparing this contribution. I am indebted to the organizers of the workshop for financial support and the Deutsche Forschungsgemeinschaft (DFG) for a travel grant. The 
works presented here were supported in part by the DFG under Grant 436 RUS 113/881/0 and the Heisenberg-Landau Programme (Grant 2008). Finally, I would like to thank S. Brodsky and G. de Téramond for attracting my attention to holographic QCD.

\section{REFERENCES}

1. A.V. Efremov, A.V. Radyushkin, Phys. Lett. B 94 (1980) 245; Theor. Math. Phys. 42 (1980) 97.

2. G.P. Lepage, S.J. Brodsky, Phys. Lett. B 87 (1979) 359; Phys. Rev. D 22 (1980) 2157.

3. V.L. Chernyak, A. R. Zhitnitsky, Phys. Rept. 112 (1984) 173.

4. A.P. Bakulev, S.V. Mikhailov, N.G. Stefanis, Phys. Lett. B 508 (2001) 279; Phys. Lett. B 590 (2004) 309, Erratum.

5. N.G. Stefanis, Eur. Phys. J. direct C 7 (1999) 1.

6. S.V. Mikhailov, A.V. Radyushkin, Sov. J. Nucl. Phys. 49 (1989) 494.

7. A.P. Bakulev, A.V. Radyushkin, Phys. Lett. B 271 (1991) 223.

8. V.Y. Petrov et al., Phys. Rev. D 59 (1999) 114018.

9. I.V. Anikin, A.E. Dorokhov, L. Tomio, Phys. Part. Nucl. 31 (2000) 509.

10. M. Praszałowicz, A. Rostworowski, Phys. Rev. D 64 (2001) 074003.

11. J. Gronberg et al., Phys. Rev. D 57 (1998) 33.

12. P. Kroll, M. Raulfs, Phys. Lett. B 387 (1996) 848.

13. A. Khodjamirian, Eur. Phys. J. C 6 (1999) 477.

14. A. Schmedding, O. Yakovlev, Phys. Rev. D 62 (2000) 116002.

15. A.P. Bakulev, S.V. Mikhailov, N.G. Stefanis, Phys. Rev. D 67 (2003) 074012.

16. A.P. Bakulev, S.V. Mikhailov, N.G. Stefanis, Phys. Lett. B 578 (2004) 91.

17. A.P. Bakulev, S.V. Mikhailov, N.G. Stefanis, Phys. Rev. D 73 (2006) 056002.

18. V.M. Braun et al., Phys. Rev. D 74 (2006) 074501.

19. M.A. Donnellan et al., PoS LATTICE2007 (2007) 369, arXiv:0710.0869 [hep-lat].
20. L. Del Debbio, Few Body Syst. 36 (2005) 77. 21. S.S. Agaev, Phys. Rev. D 72 (2005) 074020.

22. P. Ball, R. Zwicky, Phys. Lett. B 625 (2005) 225.

23. V.M. Braun, I.E. Filyanov, Z. Phys. C 44 (1989) 157.

24. H.J. Behrend et al., Z. Phys. C 49 (1991) 401.

25. B. Melić, D. Müller, K. Passek-Kumerički, Phys. Rev. D 68 (2003) 014013.

26. D.V. Shirkov, I.L. Solovtsov, Phys. Rev. Lett. 79 (1997) 1209.

27. A.I. Karanikas, N.G. Stefanis, Phys. Lett. B 504 (2001) 225.

28. N.G. Stefanis, W. Schroers, H.-C. Kim, Phys. Lett. B 449 (1999) 299.

29. N.G. Stefanis, Lect. Notes Phys. 616 (2003) 153.

30. N.G. Stefanis, W. Schroers, H.-C. Kim, Eur. Phys. J. C 18 (2000) 137.

31. A.P. Bakulev, S.V. Mikhailov, N.G. Stefanis, Phys. Rev. D 72 (2005) 074014; Phys. Rev. D 72 (2005) 119908, Erratum.

32. A.P. Bakulev, S.V. Mikhailov, N.G. Stefanis, Phys. Rev. D 75 (2007) 056005; Phys. Rev. D 75 (2007) 079901, Erratum.

33. A.P. Bakulev et al., Phys. Rev. D 70 (2004) 033014.

34. A.P. Bakulev, A.I. Karanikas, N.G. Stefanis, Phys. Rev. D 72 (2005) 074015.

35. A.P. Bakulev, arXiv:0805.0829 [hep-ph].

36. J. Volmer et al., Phys. Rev. Lett. 86 (2001) 1713.

37. C.N. Brown et al., Phys. Rev. D 8 (1973) 92.

38. J.S. Conway et al., Phys. Rev. D 39 (1989) 92.

39. A.P. Bakulev, N.G. Stefanis, O.V. Teryaev, Phys. Rev. D 76 (2007) 074032.

40. S.J. Brodsky, G.F. de Téramond, Phys. Rev. D 77 (2008) 056007.

41. S.S. Agaev, M.A. Gomshi Nobary, Phys. Rev. D 77 (2008) 074014.

42. H.R. Grigoryan, A.V. Radyushkin, Phys. Rev. D 76 (2007) 115007.

43. H.J. Kwee, R.F. Lebed, arXiv:0712.1811 [hep$\mathrm{ph}$. 